\title{
Vitamin D Status, Autonomic Nervous System Activity, and Cardiometabolic Risk
}

\author{
Johanna Lopez ${ }^{1,2,3^{*}}$, Adriana Campa', Fatma G. Huffman1, Juan P. Liuzzi'1, Tan $\mathrm{Li}^{4}$, Ana H. \\ Martinez $^{3}$, Serena M. Ferris ${ }^{3}$, Laura Lantigua ${ }^{3}$, Ashar Farooqi ${ }^{3}$, Ammar Rasul ${ }^{3}$, Steven E. Atlas ${ }^{3}$, \\ Eduard Tiozzo $^{3}$, Janet Konefal'5, Judi M. Woolger6, Herbert G. Simoes ${ }^{3,7}$, John E. Lewis ${ }^{3}$
}

\author{
${ }^{1}$ Department of Dietetics and Nutrition, Florida International University, Miami FL, USA \\ ${ }^{2}$ Jackson Memorial Hospital, Miami, FL, USA \\ ${ }^{3}$ Department of Psychiatry \& Behavioral Sciences, University of Miami Miller School of Medicine, Miami FL, USA \\ ${ }^{4}$ Department of Biostatistics, Florida International University, Miami FL, USA \\ ${ }^{5}$ Department of Family Medicine, University of Miami, Miami Miller School of Medicine, FL, USA \\ ${ }^{6}$ Department of Medicine, University of Miami Miller School of Medicine, Miami, FL, USA \\ ${ }^{7}$ Catholic University of Brasilia, Taguatinga DF, Brazil \\ Email: ^jlopez2@med.miami.edu
}

How to cite this paper: Lopez, J., Campa, A., Huffman, F.G., Liuzzi, J.P., Li, T., Martinez, A.H., Ferris, S.M., Lantigua, L., Farooqi, A., Rasul, A., Atlas, S.E., Tiozzo, E., Konefal, J., Woolger, J.M., Simoes, H.G. and Lewis, J.E. (2017) Vitamin D Status, Autonomic Nervous System Activity, and Cardiometabolic Risk. Open Journal of Internal Medicine, 7, 37-51.

https://doi.org/10.4236/ojim.2017.73004

Received: June 5, 2017

Accepted: August 6, 2017

Published: August 9, 2017

Copyright $\odot 2017$ by authors and Scientific Research Publishing Inc. This work is licensed under the Creative Commons Attribution International License (CC BY 4.0)

http://creativecommons.org/licenses/by/4.0/

(c) (i) Open Access

\begin{abstract}
Vitamin D and its association with cardiovascular disease (CVD) are currently a topic of investigation. The objective of this study was to explore the association between vitamin D status (serum 25-hydroxyvitamin D (25(OH)D)) and a cardiometabolic risk score (CMRS) derived from markers of cardiac autonomic nervous system activity, vascular dynamics, and body composition, using an innovative non-invasive technology. We found that individuals who were vitamin D sufficient $((25(\mathrm{OH}) \mathrm{D}) \geq 30 \mathrm{ng} / \mathrm{ml}, \mathrm{n}=51)$, compared to those who were vitamin $\mathrm{D}$ insufficient $(<30 \mathrm{ng} / \mathrm{ml}, \mathrm{n}=44)$, had significantly higher heart rate variability (as measured by time and frequency domain variables) and lower photoplethysmography analysis markers and CMRS. These outcomes show that vitamin D insufficient subjects had reduced cardio protective parasympathetic nervous system activity, increased endothelial dysfunction, and hence were at greater cardiometabolic risk, implying vitamin D may play a meaningful role in CVD.
\end{abstract}

\section{Keywords}

Vitamin D, Cardiometabolic Risk, Autonomic Nervous System, Endothelial Dysfunction

\section{Introduction}

Vitamin D deficiency and cardiovascular disease (CVD) are prevalent worldwide 
[1] [2]. Furthermore, the prevalence of vitamin D deficiency is high among those with CVD. Analysis of data from the National Health and Nutrition Examination Survey (NHANES) 2001-2004 showed that $68 \%$ of whites, $88 \%$ of Hispanics, and $97 \%$ of blacks with CVD also had vitamin D levels of less than $30 \mathrm{ng} / \mathrm{ml}$, as measured by 25 -hydroxyvitamin $(25(\mathrm{OH}) \mathrm{D})$ [3] [4]. Several observational studies and meta-analyses have suggested an association of vitamin $\mathrm{D}$ deficiency with CVD-related outcomes and mortality [5] [6]. These studies reported a linear, inverse relationship between CVD risk and 25(OH)D levels between 8 - 24 $\mathrm{ng} / \mathrm{ml}$ [6]. Furthermore, vitamin D supplementation demonstrated a reduction in CVD-related death, with vitamin D intake of more than 500 IU per day improving all-cause mortality, partly by decreasing CVD mortality [7] [8].

The possible relationship between vitamin $\mathrm{D}$ deficiency and CVD-related outcomes and mortality in both diseased and healthy populations is a growing area of research [2]. The most studied role of vitamin D is in regulating calcium homeostasis and mineral metabolism to maintain bone health [9]. However, vitamin D may affect multiple cellular signaling pathways that can potentially affect cardiovascular health via genomic and non-genomic actions mediated by vitamin D receptors (VDRs). These include vitamin D effects on parathyroid hormone (PTH), the renin-angiotensin-aldosterone system (RAAS), cytokine production, endothelial growth and function, and myocyte calcium influx [9].

Furthermore, vitamin D has also been shown to be involved in molecular mechanisms like biosynthesis of neurotransmitters and inflammatory signaling in areas of the central nervous system (CNS) that regulate cardiovascular activity [10] [11]. Vitamin D can permeate the blood-brain barrier and bind to VDRs located in extremely high concentrations in the midbrain and brainstem where some autonomic nervous system (ANS) neurons are located [10] [11]. Recent studies in healthy and diseased populations have found an association between vitamin D status and cardiac ANS function, with vitamin D deficiency related to cardiac ANS imbalance, more specifically with decreased parasympathetic nervous system (PNS) activity [2] [12] [13] [14]. The ANS is a branch of the peripheral nervous system that controls involuntary processes throughout the body like cardiovascular activity [15]. The cardiac ANS is composed of the stimulatory post-ganglionic sympathetic plexus of nerves and the inhibitory parasympathetic Vagus nerve, which both innervate the heart and act concomitantly to control heart rate by responding to internal and external stimuli [14] [15]. Dysfunction of cardiac ANS, more specifically of the cardioprotective vagal tone, results in an imbalance between the sympathetic nervous system (SNS) and PNS that has been well established as a risk factor for adverse cardiovascular events, such as sudden cardiac death and congestive heart failure in both healthy and diseased populations [16] [17].

Besides its effect on heart rate, ANS activity plays a role in vascular dynamics and blood pressure control, which are also influenced by endothelial function. Low vitamin D status has also been associated with endothelial dysfunction, characterized by a decreased ability to balance vasodilation and vasoconstriction. 
It is usually due to an imbalance of nitric oxide bioavailability and related to prothrombotic and proinflammatory states that increase arterial stiffness [4] [18]. Thus, vitamin D may play a role in vascular health by participating in the control of vascular tone and blood pressure via indirect ANS activity or directly affecting the endothelium. These findings suggest that vitamin D may contribute to CVD risk by orchestrating genomic and non-genomic regulatory activity at the vascular level and higher brain centers of the ANS [2] [12] [13].

Currently, the links between vitamin D status, ANS, and endothelial function have been evaluated independently, and no study has investigated the relationship between vitamin $\mathrm{D}$ and cardiometabolic risk as a composite measure that integrates various CVD risk factors such as body composition, ANS activity, and endothelial function. The purpose of this study was to investigate the association of vitamin D status with CVD risk factors and a cardiometabolic risk score (CMRS) measured using a novel, non-invasive system.

\section{Methods}

\section{Study Design and Setting}

This cross-sectional study was approved by the Institutional Review Boards (IRBs) of Florida International University (13-0390) and the University of Miami (20120195).

\section{$\underline{\text { Participants }}$}

The study enrolled 101 participants, and six were excluded due to missing data $(n=95)$. Participants were recruited from the parent study, a double-blinded randomized placebo-controlled clinical trial, which was conducted at the University of Miami. Briefly, each potential participant was screened over the phone by administering the Short Portable Mental Status Questionnaire (SPMSQ) to evaluate mental functioning (allowed up to 2 errors) and questions regarding general inclusion criteria including: (1) Men and women age 55 or older; (2) English and Spanish speakers; (3) Community-dwelling; (4) Ability to give informed consent; and (5) Participating in the parent study.

\section{Assessments}

Sociodemographic and health-related data were collected using questionnaires developed by staff of the parent study. Weight (electronic balance) and height (stadiometer) were measured and used to calculate body mass index (BMI) using the formula: body weight $(\mathrm{kg}) /$ height $\left(\mathrm{m}^{2}\right)$. Vitamin D status was measured by serum 25-hydroxyvitamin D (25(OH)D). Fasting venous blood (15 ml) was collected from every participant by a certified phlebotomist in the morning after fasting for 8 hours. The samples were sent the same day for analysis to LabCorp, which used immunochemiluminometric assays on the DiaSorin Liaison instrument to assess $25(\mathrm{OH}) \mathrm{D}$. This is a highly automated test that measures total $25(\mathrm{OH}) \mathrm{D}$, and it has been widely used by others [19].

FDA cleared and patented ANS-1 software was used to determine CMRS. This instrument combines three devices (ES-BC, TM-Oxi, and SudoPath) that use 
bioelectrical impedance, galvanic skin response, spectrophotometry, and an oscillometric blood pressure device [20] [21].

Steps of the procedure are described elsewhere and details of the devices are discussed in previous publications and the manufacturer's website

(http://www.ldteck.com) [20] [21]. The Electro-Sensor Body Composition (ESBC) device uses a single-frequency bioelectrical impedance analyzer to compute percent fat mass (\%FM) using accepted peer-reviewed published algorithms tailored to the general and obese population based on gender, height, weight, age, and activity level [20] [22].

The TM-Oxi system assesses cardiac ANS function, including both SNS and PNS, during baseline and validated cardiac autonomic reflex tests (i.e., Valsalva maneuver, deep breathing, and standing up) using an automatic oscillometric blood pressure device and pulse oximeter, which uses an optical technique (spectrophotometry) to measure the pulsatile signal or waveform, illustrated as a plethysmograph (PTG), that varies in time with each heartbeat. Thus, it assesses vascular dynamics and heart rate variability (HRV), defined as the variation in time intervals between each heart beat (i.e., R-R interval) [23]. The standard deviation of the R-R intervals (SDNN) reflects the variation between each successive heartbeat and the overall cyclic components of HRV, and it is positively correlated with left ventricular function. The square root of the mean squared differences of successive normal to normal R-R intervals (RMSSD) reflects the electrical stability of the heart, it is an indicator of PNS activity, and is usually decreased in those with chronic stress or diseased conditions. Both of these are time domain indexes of HRV [23].

The HRV analysis is also performed in frequency domains using the Fast Fourier Transform (FFT). The frequency domains are then categorized by three HRV oscillating frequencies: (1) very low frequency (VLF) associated with thermoregulation and sweating that results in oscillation in vasomotor tone controlled by SNS; (2) low frequency (LF) related to baroreflex that is under control of both SNS and PNS; and (3) high frequency (HF) associated with R-R interval changes due to breathing, under PNS control. The total power (TP) of the FFT is the sum of the areas under the curve covered by the frequency domains, it is a main indicator of ANS activity, and usually decreased in people with chronic stress or diseases. The low frequency to high frequency ratio $(\mathrm{LF} / \mathrm{HF})$ is an overall estimate of cardiac ANS balance, with a ratio $>2$ meaning SNS predominance and $<0.5$ indicating PNS predominance [21].

PTG also provides information about arterial stiffness and endothelial function. The TM-Oxi system quantifies PTG into a digital volume pulse (DVP) waveform that represents the pressure wave that spreads from the heart to the periphery (systolic peak) and reflects back to the heart (diastolic peak) during the cardiac cycle. Arterial stiffness, which is partially a result of endothelial dysfunction, is defined as the opposition to the pressure wave in the arteries, resulting in abnormal systolic and diastolic peaks. Thus, the DVP waveform is mainly 
regulated by myocardial and arterial characteristics and is used by the software to calculate indicators of vascular health and arterial status like the systemic arterial stiffness marker derived from the ascending aortic pressure waveform related to atherosclerosis (AIPTG), systemic vascular resistance (SVR), and the reflection index (RI), an indicator of stiffness of small and medium arteries [20] [21].

The data from the pulse oximeter and oscillatory blood pressure monitor are also used to assess cardiac performance. The left ventricle ejection time index (LVETi) is a measurement of the cardiac output adjusted with heart rate, the pre-ejection period (PEP) is the systolic time interval, which has been correlated with left ventricle contractility controlled by the SNS, and the ratio of these (PEP/LVETi) has been correlated with cardiac performance [20] [21].

The software integrates these measurements with demographic, anthropometric, and self-reported physical activity data to calculate a CMRS. Previous studies have shown the accuracy of these three devices, compared to standardized assessments of body composition, ANS activity, and cardiac output, and thus supporting the usefulness of these devices in detecting complications related to metabolic syndrome, diabetes, and CVD [20]. Recently, a study comparing diabetic and healthy controls showed that the CMRS calculated by this software is significantly correlated with glucose and insulin measured during an oral glucose tolerance test and was significantly higher in participants with diabetes. Also, specificity (92\%) and sensitivity (83\%) were high for the CMRS in differentiating patients with diabetes from healthy control [21].

\section{Statistical Analysis}

Using a two-tailed independent $\mathrm{t}$-test with $5 \%$ significance and $80 \%$ power and assuming an allocation ratio of 60/40 (1.5), based on published prevalence of vitamin D insufficiency in the general United States older adult population and an effect size of 0.5 for the dual task gait speed as the primary outcome variable, the total sample size calculated using G power statistical software was 134 (expected 54 vitamin D deficient and 80 vitamin D sufficient). Due to financial constraints, only 101 participants were enrolled in the study of which 6 were excluded from the analysis due to incomplete assessments and missing data.

Statistical analyses were performed on 95 participants. Data were analyzed using frequencies, percentages, ranges, means, and standard deviations. Variables were checked for non-normality using Shapiro-Wilk test, and if necessary they were transformed to achieve a normal distribution. Spearman correlations between two or more categorical variables were performed to evaluate the relationship of vitamin D status with ESC CVD risk variables and the CMRS. Independent $\mathrm{t}$-tests or chi-squares were used to compare these variables between vitamin D insufficient $(25(\mathrm{OH}) \mathrm{D}<30 \mathrm{ng} / \mathrm{ml})$ and sufficient $(\geq 30 \mathrm{ng} / \mathrm{ml})$ participants. Multiple linear regressions were used to examine the effect size and the change in CMRS expected from a one-unit change in vitamin D levels after adjusting for potential confounders. The significance level was set at $\alpha=0.05$, and statistical analyses were performed using SPSS 21. 


\section{Results}

\section{Characteristics of Participants}

The mean \pm SD $25(\mathrm{OH}) D$ level was $30.71 \pm 8.78$, with $46.3 \%(\mathrm{n}=44)$ having insufficient $(<30 \mathrm{ng} / \mathrm{ml})$ and $53.7 \%(\mathrm{n}=51)$ having sufficient $(\geq 30 \mathrm{ng} / \mathrm{ml})$ levels (Table 1). Mean \pm SD age of the participants was $63 \pm 8.8$ years, and $45 \%$ of participants were males, $45 \%$ Hispanic, $35 \%$ white Caucasian, and $16 \%$ African American. Other demographic variables are described in Table 1. Comparison of vitamin D insufficient and sufficient groups showed no significant differences between groups for age, gender, or other demographic variables except for ethnicity, with more Hispanics being vitamin D insufficient and more white Caucasians and African-Americans being vitamin D sufficient ( $P=0.008)$.

No significant differences were found between vitamin $\mathrm{D}$ insufficient and sufficient participants in number of diseases/conditions, presence of cardiovascular related diseases/disorders, over-the-counter (OTC) medication use, alcohol consumption, and smoking. However, we did find that vitamin D insufficient individuals significantly reported less prescription medication use $(P=0.021)$, fewer prescription medications $(P=0.031)$, and fewer cups of caffeinated beverages (borderline significant, $P=0.050$ ) compared to the vitamin D sufficient group.

Table 1. Characteristics of participants.

\begin{tabular}{|c|c|c|c|c|}
\hline & $\begin{array}{l}\text { Study population } \\
\qquad(\mathrm{n}=95)\end{array}$ & $\begin{array}{l}\text { Vitamin D insufficient } \\
\qquad(\mathrm{n}=44)\end{array}$ & $\begin{array}{l}\text { Vitamin D sufficient } \\
\qquad(\mathrm{n}=51)\end{array}$ & $P$ value \\
\hline Vitamin D levels $(\mathrm{ng} / \mathrm{ml})(\text { Mean } \pm S D)^{\mathrm{a}}$ & $30.71 \pm 8.78$ & $23.25 \pm 4.44$ & $37.14 \pm 6.08$ & 0.000 \\
\hline Age (years) $(\text { Mean } \pm S D)^{\mathrm{a}}$ & $62.96 \pm 6.45$ & $63.25 \pm 7.73)$ & $62.71 \pm 5.16$ & 0.693 \\
\hline Male gender $(\mathrm{N}(\%))^{\mathrm{b}}$ & $43(45.3)$ & $18(40.9)$ & $25(49.0)$ & 0.279 \\
\hline \multicolumn{5}{|l|}{ Ethnicity $(\mathrm{N}(\%))^{\mathrm{b}}$} \\
\hline Hispanic & $43(45.3)$ & $27(61.4)$ & $16(31.4)$ & \multirow{4}{*}{$0.008^{*}$} \\
\hline White Caucasian & $33(34.7)$ & $9(20.5)$ & $24(47.1)$ & \\
\hline African-American & $15(15.8)$ & 5 11.4) & $10(19.6)$ & \\
\hline Other & $4(4.2)$ & $3(6.8)$ & $1(2.0)$ & \\
\hline Number of diseases or conditions $(\text { Mean } \pm S D)^{a}$ & $4.73 \pm 3.05$ & $4.25 \pm 3.02$ & $5.14 \pm 3.04$ & 0.158 \\
\hline Participants reporting prescription medication use $(\mathrm{N}(\%))^{\mathrm{b}}$ & $71(74.7)$ & $28(63.6)$ & $43(84.3)$ & $0.021^{*}$ \\
\hline Number of prescription medications $(\text { Mean } \pm S D)^{a}$ & $2.27 \pm 2.21$ & $1.75 \pm 2.03$ & $2.73 \pm 2.28$ & $0.031^{\star}$ \\
\hline Participants reporting OTC medication use $(\mathrm{N}(\%))^{\mathrm{b}}$ & $76.0(80.0)$ & $33(75.0)$ & $43(84.3)$ & 0.258 \\
\hline Number of OTC medications $(\text { Mean } \pm \text { SD) })^{\mathrm{a}}$ & $1.47 \pm 1.12$ & $1.30 \pm 1.17$ & $1.63 \pm 1.06$ & 0.150 \\
\hline Caffeine consumers $(\mathrm{N}(\%))^{\mathrm{b}}$ & $75(78.9)$ & $33(75.0)$ & $42(82.4)$ & 0.381 \\
\hline $\begin{array}{l}\text { Cups of caffeinated coffee or caffeine } \\
\text { containing beverages per day }(\text { Mean } \pm S D)^{a}\end{array}$ & $1.64 \pm 1.38$ & $1.34 \pm 1.08$ & $1.90 \pm 1.56$ & 0.050 \\
\hline Alcoholic drinks per week $(\text { Mean } \pm S D)^{a}$ & $2.11 \pm 3.70$ & $1.82 \pm 3.51$ & $2.35 \pm 3.87$ & 0.486 \\
\hline Smokers $(\mathrm{N}(\%))^{\mathrm{b}}$ & $11.0(11.6)$ & $5(11.4)$ & $6(11.8)$ & 0.951 \\
\hline
\end{tabular}

SD: Standard Deviation; N: Number; ${ }^{\mathrm{a} I n d e p e n d e n t ~ t-t e s t s ; ~}{ }^{\text {b Chi square }}{ }^{\star} p<0.05$. 
Electro Sensor Complex CVD risk variables and the Cardiometabolic Risk Score

Body composition

The mean \pm SD weight, BMI, and $\% \mathrm{FM}$ of the participants were $76.1 \pm 17.1$ $\mathrm{kg}, 27.01 \pm 5.3 \mathrm{~kg} / \mathrm{m}^{2}$, and $30.9 \% \pm 9.1 \%$, respectively. The mean $\pm \mathrm{SD}$ systolic (SBP) and diastolic blood pressures (DBP) were $132.6 \pm 22.0$ and $76.0 \pm 14.5$ $\mathrm{mmHg}$, respectively. Body composition and blood pressure were not significantly correlated with vitamin $\mathrm{D}$ status nor different between vitamin $\mathrm{D}$ groups (Table 2).

Of the HRV time domain variables, SDNN was not significantly correlated with vitamin D status nor significantly different between vitamin D status groups, but RMSSD was significantly lower in the vitamin D insufficient group compared to the vitamin D sufficient group $(P=0.05$; Table 2$)$. Spearman correlations also showed a weak but significant positive correlation between vitamin D status and RMSSD (rho $=0.221 ; P<0.05$; Table 3 ). Of the HRV frequency domain variables, HF was significantly lower in vitamin $\mathrm{D}$ insufficient individuals compared to the vitamin D sufficient participants ( $P=0.038$; Table 2$)$, and it correlated significantly with vitamin D status (rho $=0.203 ; P<0.05$; Table 3 ). TP was also significantly different between groups, with vitamin D insufficient individuals having lower TP values compared to vitamin D sufficient participants $(P=0.045$; Table 2$)$. Although no significant difference between vitamin $\mathrm{D}$ status categories was found for the LF/HF ratio, it was weakly but significantly negatively correlated with vitamin D status ( rho $=-0.244 ; P<0.05$; Table 3).

Of the vascular dynamics markers we found that arterial stiffness (AIPTG) of the aorta and the reflection index (RI) of medium and small arteries were significantly negatively correlated with vitamin $\mathrm{D}$ status (rho $=-0.285$ and rho $=$ -0.284 ; $P$ for both $<0.01$; Table 3 ) and were significantly different between vitamin D status groups. The vitamin D insufficient group had higher AIPTG and RI values compared to the vitamin $\mathrm{D}$ sufficient group $(P=0.006$ and $P=0.006$, respectively; Table 2 ).

The PEP was significantly negatively correlated with vitamin $\mathrm{D}$ status (rho = $-0.203, P<0.05$; Table 3 ), with those who were vitamin $\mathrm{D}$ insufficient having higher PEP values than vitamin $\mathrm{D}$ sufficient individuals $(P=0.033)$. The ratio of PEP to LVETi (PEP/LVETi) was marginally significantly different $(P=0.054)$.

The mean \pm SD CMRS was $5.20 \pm 3.33$, with $48.4 \%$ having normal CMRS scores $(\leq 4)$, while $24.2 \%, 23.2 \%$, and $4.2 \%$ of the participants having mild (5 - 7), moderate $(8-10)$, and high risk of CVD $(\geq 11)$, respectively. Vitamin D status was inversely correlated with CMRS (rho $=-0.23 ; P<0.05$ ), with vitamin $\mathrm{D}$ insufficient participants having significantly higher CMRS compared with the vitamin D sufficient group ( $P=0.029)$ (Table 2 ).

Multiple linear regressions were used to evaluate the effect of vitamin D status on CMRS, after controlling for covariates $(\mathrm{F}=4.55$, d.f. $=94, P=0.013)$ (Table 4). In this fully adjusted model, CMRS would decrease by 1.79 points for those who 
Table 2. Comparison of CVD risk markers and cardiometabolic risk score (CMRS) between vitamin $\mathrm{D}$ insufficient and sufficient participants.

\begin{tabular}{|c|c|c|c|c|}
\hline Name (acronym) & $\begin{array}{c}\text { Study } \\
\text { population } \\
(\mathrm{n}=95)\end{array}$ & $\begin{array}{l}\text { Vitamin D } \\
\text { insufficient } \\
\quad(n=44)\end{array}$ & $\begin{array}{c}\text { Vitamin D } \\
\text { sufficient } \\
(\mathrm{n}=51)\end{array}$ & $P$ value \\
\hline \multicolumn{5}{|l|}{ Body composition } \\
\hline$\% \mathrm{FM}$ & $31.14 \pm 9.05$ & $32.08 \pm 9.23$ & $30.34 \pm 8.91$ & 0.354 \\
\hline Weight (kg) & $76.12 \pm 17.11$ & $73.97 \pm 15.19$ & $77.97 \pm 18.56$ & 0.257 \\
\hline BMI $\left(\mathrm{kg} / \mathrm{m}^{2}\right)$ & $27.01 \pm 5.27$ & $27.28 \pm 4.93$ & $26.77 \pm 5.58$ & 0.630 \\
\hline \multicolumn{5}{|l|}{ Blood pressure } \\
\hline $\mathrm{SBP}(\mathrm{mmHg})$ & $134.14 \pm 17.29$ & $133.05 \pm 18.60$ & $135.08 \pm 16.20$ & 0.570 \\
\hline $\mathrm{DBP}(\mathrm{mmHg})$ & $77.17 \pm 12.74$ & $76.72 \pm 12.83$ & $77.55 \pm 12.79$ & 0.756 \\
\hline \multicolumn{5}{|l|}{ HRV time domains } \\
\hline HR (bpm) & $70.00 \pm 11.06$ & $68.66 \pm 10.64$ & $71.14 \pm 11.38$ & 0.279 \\
\hline SDNN (ms) & $46.73 \pm 29.74$ & $41.70 \pm 26.70$ & $51.06 \pm 31.766$ & 0.127 \\
\hline RMSSD (ms) & $52.44 \pm 39.24$ & $43.98 \pm 33.72$ & $59.75 \pm 42.43$ & $0.05^{*}$ \\
\hline \multicolumn{5}{|c|}{ HRV frequency domains } \\
\hline $\operatorname{VLF}\left(\mathrm{ms}^{2}\right)$ & $80.92 \pm 42.44$ & $80.11 \pm 42.42$ & $81.61 \pm 42.86$ & 0.865 \\
\hline LF (n.u.) & $36.67 \pm 16.07$ & $39.75 \pm 15.30$ & $34.01 \pm 16.39$ & 0.082 \\
\hline HF (n.u.) & $35.39 \pm 17.04$ & $31.50 \pm 15.06$ & $38.74 \pm 18.05$ & $0.038^{*}$ \\
\hline LF/HF ratio & $1.57 \pm 1.62$ & $1.67 \pm 1.07$ & $1.49 \pm 1.98$ & 0.596 \\
\hline $\mathrm{TP}\left(\mathrm{ms}^{2}\right)$ & $1256.14 \pm 1702.77$ & $879.57 \pm 798.53$ & $1581.02 \pm 2161.04$ & $0.045^{*}$ \\
\hline \multicolumn{5}{|c|}{ Vascular dynamic markers } \\
\hline AIPTG (\%) & $0.514 \pm 0.22$ & $0.58 \pm 0.22$ & $0.46 \pm 0.20$ & $0.006^{*}$ \\
\hline SVR, (dyn-s-cm²) & $1423.12 \pm 347.00$ & $1464.30 \pm 406.53$ & $1387.59 \pm 285.42$ & 0.298 \\
\hline RI (\%) & $56.40 \pm 23.74$ & $63.48 \pm 24.39$ & $50.29 \pm 21.57$ & $0.006^{*}$ \\
\hline \multicolumn{5}{|l|}{ Cardiac performance } \\
\hline LVETi (ms) & $383.48 \pm 13.47$ & $382.34 \pm 14.55$ & $384.47 \pm 12.53$ & 0.445 \\
\hline PEPi (ms) & $103.19 \pm 16.43$ & $107.05 \pm 12.73$ & $99.86 \pm 18.54$ & $0.033^{*}$ \\
\hline PEPi/LVET ratio & $0.32 \pm 0.05$ & $0.33 \pm 0.04$ & $0.31 \pm 0.05$ & 0.054 \\
\hline CMRS & $5.20 \pm 3.33$ & $6.00 \pm 3.07$ & $4.51 \pm 3.41$ & $0.029^{*}$ \\
\hline
\end{tabular}

\% FM (percent fat mass), BMI (body mass index), SBP (systolic blood pressure), DBP (diastolic blood pressure), HR (heart rate), bpm (beats per minute); SDNN (standard deviation of the normal wave), ms (milliseconds), RMSSD (square root of the mean squared differences of successive normal to normal R-R intervals), VLF (very low frequency), n.u. (normalized unit), LF (low frequency), HF (high frequency), LF/HF (low frequency to high frequency), TP (total power), $\mathrm{ms}^{2}$ (milliseconds squared), AIPTG (arterial stiffness plethysmography), SVR (systemic vascular resistance), dyn-s-cm ${ }^{2}$ (dynes per square centimeter), RI (reflection index), LVETi (Left ventricle ejection time index), PEPi (pre-ejection period index), PE$\mathrm{Pi} /$ LVET (pre-ejection period index to left ventricle ejection time), CMRS (cardio-metabolic risk score); ${ }^{\star} P$ value $<0.05 ;{ }^{* *} P$ value $<0.01$. 
Table 3. Correlation between vitamin D status and CVD risk markers and cardiometabolic risk score (CMRS).

\begin{tabular}{|c|c|}
\hline & Correlation \\
\hline \multicolumn{2}{|l|}{ Body composition } \\
\hline$\% \mathrm{FM}$ & -0.131 \\
\hline Weight (kg) & 0.117 \\
\hline BMI $\left(\mathrm{kg} / \mathrm{m}^{2}\right)$ & -0.072 \\
\hline \multicolumn{2}{|l|}{ Blood pressure } \\
\hline $\mathrm{SBP}(\mathrm{mmHg})$ & 0.054 \\
\hline $\mathrm{DBP}(\mathrm{mmHg})$ & 0.032 \\
\hline \multicolumn{2}{|l|}{ HRV time domains } \\
\hline HR (bpm) & 0.089 \\
\hline SDNN (ms) & 0.187 \\
\hline RMSSD (ms) & $0.221^{*}$ \\
\hline \multicolumn{2}{|c|}{ HRV frequency domains } \\
\hline $\operatorname{VLF}\left(\mathrm{ms}^{2}\right)$ & 0.015 \\
\hline LF (n.u.) & -0.184 \\
\hline HF (n.u.) & $0.203^{*}$ \\
\hline $\mathrm{LF} / \mathrm{HF}$ ratio & $-0.244^{\star}$ \\
\hline $\mathrm{TP}\left(\mathrm{ms}^{2}\right)$ & 0.183 \\
\hline \multicolumn{2}{|c|}{ Vascular dynamic markers } \\
\hline AIPTG (\%) & $-0.285^{\star *}$ \\
\hline SVR, (dyn-s- $\left.\mathrm{cm}^{2}\right)$ & -0.063 \\
\hline RI (\%) & $-0.284^{* *}$ \\
\hline \multicolumn{2}{|l|}{ Cardiac performance } \\
\hline LVETi (ms) & 0.057 \\
\hline PEPi (ms) & $-0.203^{\star}$ \\
\hline $\mathrm{PEPi} / \mathrm{LVET}$ ratio & -0.192 \\
\hline CMRS & $-0.234^{*}$ \\
\hline
\end{tabular}

\% FM (percent fat mass), BMI (body mass index), SBP (systolic blood pressure), DBP (diastolic blood pressure), HR (heart rate), bpm (beats per minute); SDNN (standard deviation of the normal wave), ms (milliseconds), RMSSD (square root of the mean squared differences of successive normal to normal R-R intervals), VLF (very low frequency), n.u. (normalized unit), LF (low frequency), HF (high frequency), LF/HF (low frequency to high frequency), TP (total power), $\mathrm{ms}^{2}$ (milliseconds squared), AIPTG (arterial stiffness plethysmography), SVR (systemic vascular resistance), dyn-s- $\mathrm{cm}^{2}$ (dynes per square centimeter), RI (reflection index), LVETi (Left ventricle ejection time index), PEPi (pre-ejection period index), PE$\mathrm{Pi} /$ LVET (pre-ejection period index to left ventricle ejection time), CMRS (cardio-metabolic risk score); ${ }^{\star} P$ value $<0.05 ;{ }^{* *} P$ value $<0.01$.

Table 4. The relationship between vitamin D levels and CMRS in multiple linear regressions.

\begin{tabular}{|c|c|c|c|c|}
\hline & & $\beta$ (S.E.) & $P$-value & Adjusted $R^{2}$ \\
\hline Model 1 & Vitamin D status & $-1.490(0.671)$ & 0.029 & 0.04 \\
\hline \multirow{2}{*}{ Model 2} & Vitamin D status & $-1.790(0.677)$ & 0.010 & \multirow{2}{*}{0.07} \\
\hline & Number of prescription medications & $0.307(0.154)$ & 0.048 & \\
\hline
\end{tabular}

Stepwise linear regression. Dependent variable: CMRS. Model 1: Vitamin D status $(0=$ vitamin D insufficient and $1=$ vitamin D sufficient); Model 2: Model $1+$ age, gender, ethnicity, prescription medications, cups of coffee per day, alcohol use, physical activity. $\beta$ : Beta Coefficient; SE: Standard Error. 
were vitamin $\mathrm{D}$ sufficient $(\mathrm{B}(\mathrm{SE}):-1.790 \pm 0.677 ; P=0.010)$. Vitamin $\mathrm{D}$ status and number of prescription medications predicted about $7 \%$ of the variation in CMRS.

\section{Discussion}

CVD risk and vitamin D status and the benefit of vitamin D supplementation on cardiovascular health are well established [8] [24] [25]. The observations that vitamin D deficiency, cardiac ANS, and endothelial dysfunction are independently linked to CVD-related outcomes suggest a potential relationship between vitamin D deficiency and cardiovascular risk in diseased and healthy humans [9]. Vitamin D affects multiple cellular signaling pathways via genomic and nongenomic actions that have the potential to affect cardiovascular health [9]. Our results support the findings from previous studies on the association between vitamin D insufficiency and cardiometabolic risk, possibly by mediating genomic and non-genomic mechanisms that influence ANS activity and vascular function, thus affecting cardiac performance. This is the first study to investigate the relationship between vitamin D status and cardiometabolic risk, using a rapid, non-invasive method that integrates measures of ANS and endothelial function, anthropometrics, lifestyle characteristics, and body composition data.

In humans, the relationship between vitamin D status and ANS function has been studied in diseased and healthy populations. For instance, in 36 patients with non-ischemic dilated cardiomyopathy, 25(OH)D was positively correlated with HRV parameters, with vitamin D insufficiency having deleterious effects on cardiac ANS function [26]. Krause et al. [27] showed that patients with chronic kidney disease (CKD), who have impaired vitamin D synthesis, when exposed to short-term artificial sunlight heliotherapy, had a significant increase in $25(\mathrm{OH}) \mathrm{D}$ levels and an elevation in measurements of vagal activity that were severely depressed at baseline. The magnitude of $25(\mathrm{OH}) \mathrm{D}$ increase was directly correlated with the magnitude of increased cardioprotective PNS activity, implying that vitamin D levels may constitute an important role in the development of common CVD-related outcomes in this population by affecting the ANS. Chan et al. [28] reported that patients with CKD demonstrated poor cardiac ANS activity, which was characterized by decreased activity of the inhibitory PNS. Furthermore, in patients with end-stage renal disease (ESRD), depressed HRV is extremely common and usually presents as suppressed PNS activity with increased SNS input to the sino-atrial node [14] [28]. For the dissertation by Mann [14], a cross-over study was conducted in which 56 participants with ESRD on hemodialysis were randomized to either conventional $(0.25 \mathrm{mg}$ alfacalcidol 3 times per week plus placebo 3 times per week for 6 weeks) or intensive $(0.25 \mathrm{mg}$ alfacalcidol 3 times per week plus 50,000 IU ergocalciferol once a week plus placebo 2 days per week for 6 weeks) vitamin D therapy followed by a 12 -week washout period and another 6 weeks of vitamin D therapy. They did not observe significant changes in measures of cardiac autonomic tone, mineral metabolism, or RAAS activity, but an exploratory subgroup analysis found that after treatment, 
participants who remained vitamin D deficient $(25(\mathrm{OH}) \mathrm{D}<20 \mathrm{ng} / \mathrm{ml})$, compared to those who achieved sufficiency $(>30 \mathrm{ng} / \mathrm{ml}$ ), had significant ANS imbalance. In the ESRD population, abnormal HRV and ANS dysfunction are also common in this population; thus, putting them at higher risk of sudden arrhythmic death [28] [29].

In a healthy Korean population over the age of 20 years, investigators observed that $25(\mathrm{OH}) \mathrm{D}$ was positively associated with decreased HRV [30]. They observed that vitamin $\mathrm{D}$ deficient individuals $(25(\mathrm{OH}) \mathrm{D}<15 \mathrm{ng} / \mathrm{ml})$ were 3.1 times more likely to have low HRV. In 2013, Mann et al. [12] used power spectral analysis of electrocardiogram recordings to measure HRV and thus assessed cardiac ANS activity at baseline and during a graded angiotensin II challenge in 34 healthy humans. They found a significant suppression of ANS balance in vitamin $\mathrm{D}$ deficient $(25(\mathrm{OH}) \mathrm{D}<20 \mathrm{ng} / \mathrm{ml})$ participants at baseline, but no difference was observed during the challenge. In an intervention study, the same group demonstrated that healthy adults supplemented with 10,000 IU of vitamin $\mathrm{D}$ for four weeks had a significant improvement in cardiac ANS balance, specifically in enhancing the cardio-protective PNS activity [13]. In our study, we found that vitamin D insufficient participants had significantly lower RMSSD, $\mathrm{HF}$, and TP values, all of which are indicators of PNS activity meaning that vitamin D insufficient participants had less cardioprotective PNS activity during the cardiac autonomic tests.

Besides the link between vitamin D and ANS, 25(OH)D levels have been independently associated with several tests of endothelial dysfunction, arterial stiffness, and coronary flow reserve [31]. In addition, studies have demonstrated that vitamin $\mathrm{D}$ treatment improves arterial stiffness as measured by pulse wave velocity [32]. In our study, we found that vitamin D insufficient participants had significantly increased stiffness of the aorta (AIPTG) and the medium and small arteries (RI). Thus, vitamin D may affect vascular tone indirectly via modulation of cardiac ANS activity or directly by affecting endothelial function. Furthermore, we found that vitamin D insufficient subjects had increased SNS activity as measured by the PEP. A higher PEP is inversely correlated with left ventricular contraction, and thus vitamin D insufficient participants may have impaired cardiac performance compared to vitamin D sufficient subjects. Our study, like other cross-sectional and longitudinal studies, supports a relationship between low vitamin D metabolites and cardiovascular risk [9]. We found that the overall CMRS score was inversely correlated with vitamin D status, with vitamin D insufficient participants having higher CMRS, and thus being at higher risk of cardiometabolic events.

Our study found that compared to vitamin D sufficient subjects, those who were vitamin D insufficient had lower PNS activity, increased arterial stiffness, and depressed cardiac performance and thus were at higher risk of cardiometabolic risk as shown by a significantly lower CMRS, calculated from composite measures of body composition, ANS activity, and endothelial function by using a novel system. These data suggest that vitamin $\mathrm{D}$ may play a significant role in 
CVD, probably through modulation of cardiac performance by affecting ANS activity, vascular tone, and endothelial function.

Due to the nature of this cross-sectional study, we cannot conclude that vitamin D insufficiency is a cause of increased cardiovascular risk. Furthermore, vitamin $\mathrm{D}$ is part of complex mineral metabolism functions that involve factors known to influence cardiovascular risk, including PTH, phosphate, and calcium. Our study did not measure any of these potential intermediaries between vitamin D and cardiac health nor assess biomarkers that are clinically established as risk factors for CVD, such as cholesterol, triglycerides, glucose, hemoglobin A1C, and others. Thus, future prospective randomized controlled intervention studies that use cardiovascular events as a primary outcome and measure other biomarkers related to vitamin $\mathrm{D}$, mineral metabolism, and cardiovascular outcomes are warranted to examine the possible mechanism by which this nutrient may affect cardiovascular health and evaluate the benefit of supplementation on cardiometabolic risk in vitamin $\mathrm{D}$ insufficient older adults.

Vitamin D supplementation may be a cost-effective treatment to improve ANS and endothelial function in healthy and chronic disease populations and thus reduce CVD risk. Based on the acceptance of preliminary findings on the effect of supplementation on CVD, a cost-benefit analysis has estimated a reduction of as many as 336,000 deaths per year, including 180,000 deaths from CVD, if the United States adult population were to raise vitamin D levels above 40 $\mathrm{ng} / \mathrm{ml}$. This also translates into a reduction of about $\$ 130$ billion per year in direct healthcare costs [33].

\section{Acknowledgements}

We are thankful to all of the volunteers who participated in this study. We also want to thank the LD Technology for lending the equipment used for data collection and providing training and support in using the software.

\section{Compliance with Ethical Standards}

Conflicts of interest. The authors do not have any conflicts of interest.

Ethical Approval: All procedures followed were in accordance with the ethical standards of the University of Miami and Florida International University Instiutional Review Boards and with the Helskinki Declaration of 1975, as revised in 2000. Informed consent was obtained from all study participants. No animal studies were carried out by the authors for this article.

\section{Source of Funding}

This study was a cross-sectional analysis of the baseline data of the parent interventional study supported by Biotics Research Corporation. The sponsor had no role in the design and the conduct of the study; in the collection, analysis, and interpretation of the data; in the preparation of the manuscript; or in the review or approval of the manuscript. Any opinions, findings, and conclusions or rec- 
ommendations expressed in this material are solely the responsibility of the authors and do not necessarily represent the official views of the sponsor.

\section{References}

[1] Holick, M.F. (2007) Vitamin D Deficiency. The New England Journal of Medicine, 357, 266-281. https://doi.org/10.1056/NEJMra070553

[2] Mann, M.C., Hollenberg, M.D. and Hanley, D.A. (2015) Vitamin D, the Autonomic Nervous System, and Cardiovascular Risk. Physiological Reports, 3, 123491 1234914. https://doi.org/10.14814/phy2.12349

[3] Kim, D.H., Sabour, S. and Sagar, U.N. (2008) Prevalence of Hypovitaminosis D in Cardiovascular Diseases (from the National Health and Nutrition Examination Survey 2001 to 2004). The American Journal of Cardiology, 102, 1540-1544. https://doi.org/10.1016/j.amjcard.2008.06.067

[4] Norman, P.E. and Powell, J.T. (2014) Vitamin D and Cardiovascular Disease. Circulation Research, 114, 379-393. https://doi.org/10.1161/CIRCRESAHA.113.301241

[5] Schottker, B., Jorde, R. and Peasey, A. (2014) Vitamin D and Mortality: MetaAnalysis of Individual Participant Data from a Large Consortium of Cohort Studies from Europe and the United States. BMJ, 348, 1-15. https://doi.org/10.1136/bmj.g3656

[6] Wang, L., Song, Y. and Manson, J.E. (2012) Circulating 25-Hydroxy-Vitamin D and Risk of Cardiovascular Disease: A Meta-Analysis of Prospective Studies. Circulation: Cardiovascular Quality and Outcomes, 5, 819-829. https://doi.org/10.1161/CIRCOUTCOMES.112.967604

[7] Parker, J., Hashmi, O. and Dutton, D. (2010) Levels of Vitamin D and Cardiometabolic Disorders: Systematic Review and Meta-Analysis. Maturitas, 65, 225-236. https://doi.org/10.1016/j.maturitas.2009.12.013

[8] Wang, L., Manson, J.E. and Song, Y. (2010) Systematic Review: Vitamin D and Calcium Supplementation in Prevention of Cardiovascular Events. Annals of Internal Medicine, 152, 315-323. https://doi.org/10.7326/0003-4819-152-5-201003020-00010

[9] Beveridge, L.A. and Witham, M.D. (2013) Vitamin D and the Cardiovascular System. Osteoporosis International, 24, 2167-2180. https://doi.org/10.1007/s00198-013-2281-1

[10] DeLuca, G.C., Kimball, S.M. and Kolasinski, J. (2013) Review: The Role of Vitamin D in Nervous System Health and Disease. Neuropathology and Applied Neurobiology, 39, 458-484. https://doi.org/10.1111/nan.12020

[11] Garcion, E., Wion-Barbot, N. and Montero-Menei, C.N. (2002) New Clues about Vitamin D Functions in the Nervous System. Trends in Endocrinology and Metabolism: TEM, 13, 100-105. https://doi.org/10.1016/S1043-2760(01)00547-1

[12] Mann, M.C., Exner, D.V. and Hemmelgarn, B.R. (2013) Vitamin D Levels Are Associated with Cardiac Autonomic Activity in Healthy Humans. Nutrients, 5, 2114 2127. https://doi.org/10.3390/nu5062114

[13] Mann, M.C., Exner, D.V. and Hemmelgarn, B.R. (2014) Vitamin D Supplementation Is Associated with Improved Modulation of Cardiac Autonomic Tone in Healthy Humans. International Journal of Cardiology, 172, 506-508. https://doi.org/10.1016/j.ijcard.2014.01.058

[14] Mann, M.C. (2015) Vitamin D and Cardiac Autonomic Tone: Cardiovascular Implications in Humans with and without Chronic Kidney Disease. University of Calgary, Calgary. 
[15] Kapa, S., Venkatachalam, K.L. and Asirvatham, S.J. (2010) The Autonomic Nervous System in Cardiac Electrophysiology: An Elegant Interaction and Emerging Concepts. Cardiology in Review, 18, 275-284. https://doi.org/10.1097/CRD.0b013e3181ebb152

[16] Goldberger, J.J., Cain, M.E. and Hohnloser, S.H. (2008) American Heart Association/American College of Cardiology Foundation/Heart Rhythm Society: Scientific Statement on Noninvasive Risk Stratification Techniques for Identifying Patients at Risk for Sudden Cardiac Death: A Scientific Statement from the American Heart Association Council on Clinical Cardiology Committee on Electrocardiography and Arrhythmias and Council on Epidemiology and Prevention. Circulation, 118, 14971518. https://doi.org/10.1161/CIRCULATIONAHA.107.189375

[17] Lauer, M.S. (2009) Autonomic Function and Prognosis. Cleveland Clinic Journal of Medicine, 76, S18-S22. https://doi.org/10.3949/ccjm.76.s2.04

[18] Cozzolino, M., Ketteler, M. and Zehnder, D. (2010) The Vitamin D System: A Crosstalk between the Heart and Kidney. European Journal of Heart Failure, 12, 1031-1041. https://doi.org/10.1093/eurihf/hfq112

[19] Wagner, D., Hanwell, H.E. and Vieth, R. (2009) An Evaluation of Automated Methods for Measurement of Serum 25-Hydroxyvitamin D. Clinical Biochemistry, 42, 1549-1556. https://doi.org/10.1016/j.clinbiochem.2009.07.013

[20] Lewis, J.E., Tannenbaum, S.L. and Gao, J. (2011) Comparing the Accuracy of ESBC, EIS-GS, and ES Oxi on Body Composition, Autonomic Nervous System Activity, and Cardiac Output to Standardized Assessments. Medical Devices, 4, 169-177. https://doi.org/10.2147/MDER.S24291

[21] Lewis, J.E., Lantigua, L. and Atlas, S.E. (2014) A Cross-Sectional Assessment to Detect Type 2 Diabetes with Endothelial and Autonomic Nervous System Markers Using a Novel System. Journal of Diabetes and Metabolic Disorders, 13, 118-125. https://doi.org/10.1186/s40200-014-0118-x

[22] Chumlea, W.C., Guo, S.S. and Kuczmarski, R.J. (2002) Body Composition Estimates from NHANES III Bioelectrical Impedance Data. International Journal of Obesity and Related Metabolic Disorders. Journal of the International Association for the Study of Obesity, 26, 1596-1609. https://doi.org/10.1038/sj.ijo.0802167

[23] Task Force of the European Society of Cardiology and the North American Society of Pacing and Electrophysiology (1996) Heart Rate Variability: Standards of Measurement, Physiological Interpretation and Clinical Use. Circulation, 93, 1043-1065. https://doi.org/10.1161/01.CIR.93.5.1043

[24] Wang, T.J., Pencina, M.J. and Booth, S.L. (2008) Vitamin D Deficiency and Risk of Cardiovascular Disease. Circulation, 117, 503-511. https://doi.org/10.1161/CIRCULATIONAHA.107.706127

[25] Autier, P. and Gandini, S. (2007) Vitamin D Supplementation and Total Mortality: A Meta-Analysis of Randomized Controlled Trials. Archives of Internal Medicine, 167, 1730-1737. https://doi.org/10.1001/archinte.167.16.1730

[26] Cetin, M., Kozdag, G. and Ural, D. (2014) Could Decreased Vitamin D Levels Be Related with Impaired Cardiac Autonomic Functions in Patients with Chronic Heart Failure: An Observational Study. The Anatolian Journal of Cardiology, 14, 434-441. https://doi.org/10.5152/akd.2014.4869

[27] Krause, M., Anschutz, W. and Vettorazzi, E. (2014) Vitamin D Deficiency Intensifies Deterioration of Risk Factors, Such as Male Sex and Absence of Vision, Leading to Increased Postural Body Sway. Gait \& Posture, 39, 166-171. https://doi.org/10.1016/j.gaitpost.2013.06.017 
[28] Chan, C.T., Levin, N.W. and Chertow, G.M. (2010) Determinants of Cardiac Autonomic Dysfunction in ESRD. Clinical Journal of the American Society of Nephrology: CJASN, 5, 1821-1827. https://doi.org/10.2215/CJN.03080410

[29] Chan, C.T. (2008) Heart Rate Variability in Patients with End-Stage Renal Disease: An Emerging Predictive Tool for Sudden Cardiac Death? Nephrology, Dialysis, Transplantation: Official Publication of the European Dialysis and Transplant Association-European Renal Association, 23, 3061-3062.

https://doi.org/10.1093/ndt/gfn280

[30] Tak, Y.J., Lee, J.G. and Kim, Y.J. (2014) 25-Hydroxyvitamin D and Its Relationship with Autonomic Dysfunction Using Time- and Frequency-Domain Parameters of Heart Rate Variability in Korean Populations: A Cross-Sectional Study. Nutrients, 6, 4373-4388. https://doi.org/10.3390/nu6104373

[31] Al-Mheid, I., Patel, R. and Murrow, J. (2011) Vitamin D Status Is Associated with Arterial Stiffness and Vascular Dysfunction in Healthy Humans. Journal of the American College of Cardiology, 58, 186-192. https://doi.org/10.1016/j.jacc.2011.02.051

[32] Breslavsky, A., Frand, J. and Matas, Z. (2013) Effect of High Doses of Vitamin D on Arterial Properties, Adiponectin, Leptin and Glucose Homeostasis in Type 2 Diabetic Patients. Clinical Nutrition (Edinburgh, Scotland), 32, 970-975. https://doi.org/10.1016/j.clnu.2013.01.020

[33] Baggerly, C.A., Cuomo, R.E. and French, C.B. (2015) Sunlight and Vitamin D: Necessary for Public Health. Journal of the American College of Nutrition, 34, 359365. https://doi.org/10.1080/07315724.2015.1039866

Submit or recommend next manuscript to SCIRP and we will provide best service for you:

Accepting pre-submission inquiries through Email, Facebook, LinkedIn, Twitter, etc. A wide selection of journals (inclusive of 9 subjects, more than 200 journals)

Providing 24-hour high-quality service

User-friendly online submission system

Fair and swift peer-review system

Efficient typesetting and proofreading procedure

Display of the result of downloads and visits, as well as the number of cited articles

Maximum dissemination of your research work

Submit your manuscript at: http://papersubmission.scirp.org/

Or contact ojim@scirp.org 